\title{
THEOREMS OF POINTS AND PLANES IN THREE-DIMENSIONAL PROJECTIVE SPACE
}

\author{
DAVID G. GLYNN
}

\author{
(Received 15 July 2007; accepted 4 April 2008)
}

\author{
Communicated by L. M. Batten
}

\begin{abstract}
We discuss $n_{4}$ configurations of $n$ points and $n$ planes in three-dimensional projective space. These have four points on each plane, and four planes through each point. When the last of the $4 n$ incidences between points and planes happens as a consequence of the preceding $4 n-1$ the configuration is called a 'theorem'. Using a graph-theoretic search algorithm we find that there are two $8_{4}$ and one $9_{4}$ 'theorems'. One of these $8_{4}$ 'theorems' was already found by Möbius in 1828, while the $9_{4}$ 'theorem' is related to Desargues' ten-point configuration. We prove these 'theorems' by various methods, and connect them with other questions, such as forbidden minors in graph theory, and sets of electrons that are energy minimal.
\end{abstract}

2000 Mathematics subject classification: primary 51A20; secondary 51A45, 05B25, $05 \mathrm{~B} 35$.

Keywords and phrases: geometry, configuration, projective space, theorem, minimal energy electrons, forbidden minor, Fano, Pappus, Desargues, Möbius.

\section{Introduction}

DEFINITION 1.1. A (combinatorial) $n_{k}$ configuration is an incidence structure $(P, B, I)$ with a set $P$ of $n$ points and a set $B$ of $n$ blocks (which may be considered to be subsets of $P$ ), such that each block contains $k$ of the points, and each point is on $k$ of the blocks. A point $p \in P$ is on a block $b \in B$ if and only if $p I b$, where $I$ is the incidence relation. Note that we usually omit the term 'combinatorial'. When the points are given homogeneous coordinates of a certain $(k-1)$-dimensional projective space so that the blocks are on hyperplanes (with dual coordinates), we say that the $n_{k}$ configuration is 'embedded'.

In general, $n_{k}$ 'theorems' are combinatorial $n_{k}$ configurations, with the property that whenever they are embedded in $(k-1)$-dimensional projective space, with $k$ points on each hyperplane and $k$ hyperplanes through each point, the last of the $k n$ incidences is determined by the first $k n-1$. Sometimes this occurs only when the ambient space is

(C) 2010 Australian Mathematical Publishing Association Inc. 1446-7887/2010 \$16.00 
coordinatised by a certain field, or it may be that the 'theorem' is valid for all projective spaces. For some background to finite projective geometry see [8].

Definition 1.2 (Glynn [10], Levi [13]). The Levi graph of an $n_{k}$ configuration $C$ with point-set $P$ and block-set $B$ is the bipartite graph $(P \cup B, E)$, where $\{p, b\}$ is an edge in $E$ if and only if $p \in P, b \in B$, and $p$ is incident with $b$; that is, $(p, b)$ is a flag of $C$.

REMARK. The generation of $n_{k}$ configurations via graphical methods uses the fact that a graph is the Levi graph of an $n_{k}$ configuration if and only if it has $2 n$ vertices, is bipartite and is regular of degree $k$.

A disconnected configuration could be split into a number of connected configurations, which may be dealt with separately. Also, in $(k-1)$-dimensional space, $k-2$ general points generate a secundum, that is, a $(k-3)$-dimensional subspace, while $k-1$ points generate a hyperplane, and dually $k-2$ hyperplanes intersect in a line, while $k-1$ hyperplanes intersect in a point. Thus, to avoid an embedded $n_{k}$ configuration having repeated points, hyperplanes or other degeneracy we note the following.

REMARK. In order to exclude 'degenerate' cases we assume that:

- the configuration (or, equivalently, its Levi graph) is connected;

- $\quad$ no pair of distinct points are incident with the same $k-1$ blocks; and

- no pair of distinct blocks are incident with the same $k-1$ points.

This paper provides details of certain $n_{4}$ 'theorems', which are not found in Baker [1], Hilbert [11, 12], or in other easily obtainable sources. These 'theorems' were found using the embedding algorithm of Glynn [10], implemented using a short program in MAGMA [2], with an input of all connected bipartite graphs on $2 n$ vertices that are regular of degree $k$. These graphs were created using the geng (generate graph) program of McKay's NAUTY package [14].

Since the general philosophy and theories of $n_{k}$ 'theorems' are given by Glynn [10], we concentrate on the properties of the $8_{4}$ and $9_{4}$ 'theorems' found by searching when $n$ is small.

It was shown in [10] that the embedding problem reduces to finding a rooted spanning tree of the related Levi graph, and this tree can easily be found by using a well-known greedy algorithm. A single algebraic equation can be found involving many free parameters that expresses the embedding condition. Although a 'standard' embedding might not exist for a given field, if the base field is chosen large enough (for example, if it is algebraically closed) and with the right characteristic, such embeddings should usually appear, although in some cases it can be shown that embeddings (with all of the points and hyperplanes distinct) can never appear. (One of the $10_{3}$ configurations is a case in point.) The 'spanning tree algorithm' generalizes and explains results for $n_{3}$ configurations for which various methods have already been found. 
DEFINITION 1.3. A closed $n_{k}$ configuration is one for which the last incidence (for example, using the spanning tree algorithm) is always trivially satisfied. Naturally, this might depend on the field that is chosen. If this holds we call it a 'theorem'.

DEFINITION 1.4. The complement of an $n_{k}$ configuration $K$ with Levi graph $L$ is the $n_{n-k}$ configuration $\bar{K}$ with complementary bipartite Levi graph $\bar{L}$. (The flags of $K$ are replaced by antiflags, and vice versa. Equivalently, the edges of $L$ are deleted, and where there is no edge between a point and block an edge is inserted.)

The complement of any closed (standard) configuration ('theorem') was shown by Glynn [10] to be closed, so that, for example, the well-known Pappus $9_{3}$ 'theorem' in a plane over a field corresponds to a 96 'theorem' in five-dimensional space over the same field (actually lying on a Veronese surface).

In some important cases, however, the complement of a 'theorem' degenerates by having all of its hyperplanes passing through a common point. (Dually, the points would lie in a hyperplane.) In particular this happens when the $n_{k}$ configuration comes by projection from a similar configuration in one higher dimension; see [10]. The $10_{3}$ and 94 'theorems' have this property and this is why we have not included the complementary $10_{7}$ and $9_{5}$ 'theorems' in the list of nondegenerate 'theorems' in Section 9.

One of the configurations investigated has parameters $8_{4}$, and is a 'theorem' when embedded in any three-dimensional space coordinatised by a field. This is similar to the well-known Möbius 84 'theorem', but it is not the same. Another configuration has parameters $9_{4}$, and it can be shown to be a 'theorem' for any three-dimensional projective space, by using the two triangle $10_{3}$ configurational 'theorem' of Desargues. Some other similarities with Desargues' theorem are discussed in this paper. There is a connection betwen $n_{k}$ 'theorems' and forbidden minors in graph and matroid theory. In physics, the 84 and 94 'theorems' are attained by sets of eight and nine electrons on a sphere that are energy minimal. Models of the configurations are also discussed in Section 8 .

\section{Embedding configurations into projective space}

Several methods were discussed in [10] to embed an $n_{k}$ configuration into projective space. Here we progress to a 'spanning tree' algorithm that generalizes all of them.

DEFINITION 2.1. A spanning tree of a connected graph with $v$ vertices is a set of $v-1$ edges containing no circuit: thus, each vertex appears on at least one of the edges of the tree.

To embed a configuration $K$ into projective space one must assign homogeneous coordinates to each point and dual coordinates to each block (considered as a hyperplane) of $K$ so that the incidences between these points and hyperplanes in space mirror those of $K$.

We spell out the method to embed an $n_{k}$ configuration using a spanning tree of the Levi graph; see Definitions 1.2 and 2.1. What one does is start from the outside of the tree (that is, with vertices of valency one), and work inwards, always keeping a 
connected tree by deleting vertices of valency one. This gives a construction sequence of elements (points and hyperplanes) of the configuration, so that the homogeneous coordinates of the first (or outer) elements of the tree are known before the later elements. The final incidence is problematic (depending, in general, on the solution of an algebraic equation) unless of course the configuration is a 'theorem'.

\section{The search algorithm}

Here we explain how a computer search was made for $n_{k}$ 'theorems'. The method relied on using certain popular computer packages: the general mathematical program MAGMA [2] and the graph-theoretic program NAUTY [14]. The algorithm was easily implemented using a MAGMA script, since that program has inbuilt functions that reference NAUTY. We wrote it so that all calculations were made within the geometry over the field of rational numbers $\mathbb{Q}$. It could easily be extended to other fields, in particular fields of characteristic $p$. Doing this over the reals, for example, could create problems in that determinants calculated on a computer have only a certain precision.

3.1. The method. Our method is as follows.

Step 1. Construct all nonisomorphic, connected regular bipartite graphs of $2 n$ vertices and of valency $k$. These are easily seen to be in correspondence with the Levi graphs of combinatorial connected $n_{k}$ configurations. We used the 'geng', or 'generate graph', function of NAUTY.

Step 2. For each of these graphs $G$ construct a spanning tree $S$. This will have all $2 n$ vertices of $G$ and $2 n-1$ edges without any circuit.

Step 3. Find a vertex $v$ of $S$ of valency one. (It is on the 'outside' of $S$.)

Step 4. Construct the orthogonal space $O(v)$ in $k$-dimensional vector space $\mathbb{Q}^{k}$ to the space generated by the coordinates of all vertices in $G$ joined to $v$ that have already been given coordinates. There are at most $k-1$ of these (because $S$ has more vertices connected to $v$ ), and so the rank of $O(v)$ is at least one.

Step 5. Find a random vector $c(v)$ in $O(v)$ and use this as the coordinates for $v$. (Here $v$ is either a point or hyperplane of $\operatorname{PG}(k-1, \mathbb{Q})$ and so $c(v)$ gives either the homogeneous coordinates or dual homogeneous coordinates, respectively, for $v$.)

Step 6. Delete $v$ from $S$ and repeat the loop starting with Step 3 until there is only one vertex left in $S$.

Step 7. At this stage all points and hyperplanes except one in the $n_{k}$ configuration have been given coordinates. So, for the last vertex $v$ of $S$ determine whether $\operatorname{rank}(O(v))$ is zero or positive. If $O(v)$ has rank zero then $G$ does not correspond to an $n_{k}$ 'theorem'. However, if the rank is one (in general, it should not be more than that), then it almost certainly is an $n_{k}$ 'theorem'. (Analogously, it is highly likely that a set of $k$ vectors in $\mathbb{Q}^{k}$, randomly chosen by a computer, is independent). 


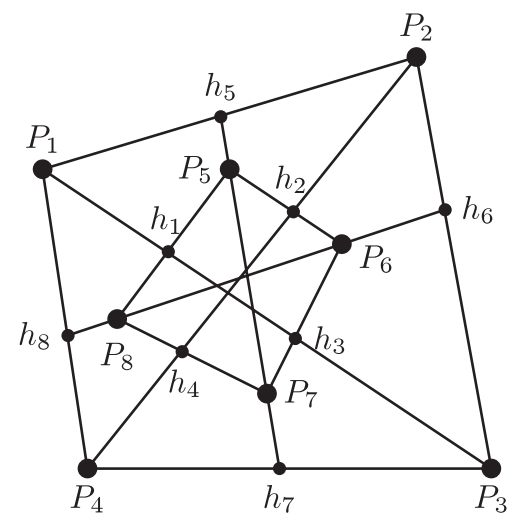

FIGURE 1. The 84 'theorem'.

Step 8. To be doubly sure, it is possible to repeat the algorithm again with different random coordinates. If the rank of the last space $O(v)$ is positive, then we are highly optimistic that we have an $n_{k}$ 'theorem'.

3.2. The results of the search. The algorithm of Section 3.1 was performed on a small computer for various parameters $n_{k}$ with $n \leq 11$ and with the field set as the rational numbers. It found the known $n_{k}$ 'theorems' such as the Pappus $9_{3}$, Desargues $10_{3}$ and Möbius $8_{4}$ configurations very quickly but also determined that two other configurations, $8_{4}$ and 94 , were strong candidates for being 'theorems' as well. (Some other configurations were rejected even though they passed the above algorithm, because they had degenerate properties.) We consider these $8_{4}$ and $9_{4}$ configurations in the following sections.

\section{An 84 'theorem' for $\operatorname{PG}(3, \mathbb{F}), \mathbb{F}$ a field}

4.1. The description of the $8_{4}$ configuration. Note that an $n_{4}$ configuration will have $n$ points and $n$ planes in three-dimensional space as in this case hyperplanes are the same as planes. Here we describe the non-Möbius 84 configuration found by the computer.

Consider Figure 1. There are eight 'large' points, in two subsets which are tetrahedra in three-dimensional space. Label the first tetrahedron $P_{1}, P_{2}, P_{3}, P_{4}$, and the second tetrahedron $P_{5}, P_{6}, P_{7}, P_{8}$. There are also eight 'small' points $h_{1}, h_{2}, h_{3}$, $h_{4}, h_{5}, h_{6}, h_{7}, h_{8}$, which are in correspondence with the planes of the configuration. Thus, it can be considered to be two tetrahedra. Each tetrahedron has six edges, two of which are special (we call them 'diagonals') and they are skew. The remaining four edges form a 4-cycle. Each plane of the 84 configuration contains an edge of one tetrahedron and a diagonal of the other tetrahedron: thus, it contains four points in total. The diagonals of the first tetrahedron are the line joining $P_{1}$ and $P_{3}$ and the line joining $P_{2}$ and $P_{4}$. Similarly, the diagonals of the second tetrahedron connect $P_{5}$ with $P_{7}$, and $P_{6}$ with $P_{8}$. 
Thus, we assume that the planes of the configuration are:

$$
\begin{array}{ll}
h_{1}:=\left\{P_{1}, P_{3}, P_{5}, P_{8}\right\}, \quad h_{2}:=\left\{P_{2}, P_{4}, P_{5}, P_{6}\right\}, \\
h_{3}:=\left\{P_{1}, P_{3}, P_{6}, P_{7}\right\}, \quad h_{4}:=\left\{P_{2}, P_{4}, P_{7}, P_{8}\right\}, \\
h_{5}:=\left\{P_{1}, P_{2}, P_{5}, P_{7}\right\}, \quad h_{6}:=\left\{P_{2}, P_{3}, P_{6}, P_{8}\right\}, \\
h_{7}:=\left\{P_{3}, P_{4}, P_{5}, P_{7}\right\}, \quad h_{8}:=\left\{P_{1}, P_{4}, P_{6}, P_{8}\right\} .
\end{array}
$$

Each plane $h_{i}$ contains four points $P_{j}$ and within this plane, the edge of one tetrahedron meets the diagonal of the other in a certain point. If we identify the planes with the small points $h_{i}$ of the diagram, then there are four lines each containing four points in three-dimensional space. These are formed from the diagonals of the two tetrahedra:

$$
\begin{array}{ll}
l_{1}:=\left\{P_{1}, P_{3}, h_{1}, h_{3}\right\}, & l_{2}:=\left\{P_{2}, P_{4}, h_{2}, h_{4}\right\}, \\
l_{3}:=\left\{P_{5}, P_{7}, h_{5}, h_{7}\right\}, \quad l_{4}:=\left\{P_{6}, P_{8}, h_{6}, h_{8}\right\} .
\end{array}
$$

There are eight three-point lines (which are the nondiagonal edges of the tetrahedra):

$$
\begin{array}{llll}
\left\{P_{1}, P_{2}, h_{5}\right\}, & \left\{P_{2}, P_{3}, h_{6}\right\}, & \left\{P_{3}, P_{4}, h_{7}\right\}, & \left\{P_{4}, P_{1}, h_{8}\right\}, \\
\left\{P_{5}, P_{6}, h_{2}\right\}, & \left\{P_{6}, P_{7}, h_{3}\right\}, & \left\{P_{7}, P_{8}, h_{4}\right\}, & \left\{P_{8}, P_{5}, h_{1}\right\} .
\end{array}
$$

REMARK. It is an easy exercise to see that the above 84 configuration is self-dual; that is, the transpose of any incidence matrix between points and hyperplanes is isomorphic to the incidence matrix. Also, it is self-complementary; that is, the complement of any incidence matrix is isomorphic to itself.

REMARK. Using NAUTY [14] we calculated the order of the automorphism group of the Levi graph of this 84 configuration, and it is 64 . Since this configuration is selfdual, the configuration has an automorphism group $G$ of size 32. Let each point $P_{i}$ of the 84 configuration be represented as $i$. Thus we can consider $G$ as a subgroup of the symmetric group $S_{8}$ of permutations of the set $\{1, \ldots, 8\}$. Using MAGMA [2] we found that

$$
G=\langle(18)(45)(36)(27),(13)(56)(78),(57)(68)\rangle
$$

( $G$ also acts equivalently on the eight points $h_{i}$, which form an isomorphic $8_{4}$ configuration). The centre of $G$ is $\langle(13)(24)(57)(68)\rangle$ of order two, which flips the points on the diagonals of each tetrahedron. There are automorphisms of order eight in $G$, for example, $(1,7,2,8,3,5,4,6)$, so the group is transitive on the points. Note also that (57)(68) fixes the first tetrahedron pointwise. In contrast, the 84 configuration of Möbius [15] has an automorphism group of size 192. See the following.

REMARK. An easy way to remember how to construct the block patterns of the Möbius $8_{4}$ configuration and the 'other' $8_{4}$ configuration is as follows. For the Möbius 84 configuration the points are on two 'squares', the corresponding sides of which are 'parallel'. The eight blocks are formed by taking any two points on a side of one square together with two points on a parallel side of the other square. The 'other' 
84 configuration also has two 'squares' of points, but in this case each diagonal of one square is parallel to two sides of the other square. Then each of the eight blocks is formed by two points on a side of one square joined with the two points on the diagonal of the other square that is parallel to that side. Note that the squares in these constructions do not correspond to blocks, and so will not form planes in a general three-dimensional representation.

REMARK. The group of order 192 of the Möbius 84 configuration may be constructed as a subgroup of index $15 \times 7=105$ in the simple group $\operatorname{PGL}(4,2)$. We may consider an incident point/plane pair $(P, \pi)$, that is, a 'flag' in the finite projective space $\mathrm{PG}(3,2)$ of dimension three over the finite field $\mathrm{GF}(2)$ of order two. The structure consisting of the eight points of $\mathrm{PG}(3,2)$ not on $\pi$ and the eight planes of $\mathrm{PG}(3,2)$ not through $P$ is the Möbius 84 configuration. However, the subgroup $S(P, \pi)$ of the automorphism group $\operatorname{PGL}(4,2)$ of $\operatorname{PG}(3,2)$ that fixes the flag contains the normal subgroup $T(\pi)$ (see the next remark) which are the translations with axis $\pi$ (this is isomorphic to the elementary abelian group $Z_{2}^{3}$ of order eight). Next, the factor group of $S(P, \pi) / T(\pi) \cong S_{4}$, which may be considered to be the subgroup of index seven in $\operatorname{PGL}(3,2)$, permuting the four lines not through $P$ in the plane $\pi$. Thus, we obtain the structure of the group of order $192=24 \times 8$.

REMARK. Felix Klein once noted that the way to obtain a normal subgroup $N$ in the automorphism group $G$ of a geometrical structure is first to find a set $S$ of geometrical objects related to the structure that are permuted amongst themselves. Then, $N$ is the subgroup of $G$ that fixes each $s$ in $S$. For example, in an affine geometry the parallel classes are permuted amongst themselves. The set of translations, which are the automorphisms that fix all parallel classes, therefore forms a normal subgroup of the automorphism group of the affine geometry.

REMARK. The automorphism group of the Möbius $8_{4}$ configuration, considered as a permutation group on the points, is imprimitive: each point $X$ has a unique 'antipodal' point $X^{\prime}$, such that no planes of the configuration pass through both $X$ and $X^{\prime}$. Thus, there are four sets of imprimitivity, each of size two. Similarly, the automorphism group of the other $8_{4}$ configuration is imprimitive: there are two sets of imprimitivity of size four, which are the two tetrahedra considered previously.

There are alternative proofs, geometric and algebraic, of the fact that the 'other' $8_{4}$ configuration defined above is a 'theorem'. All proofs rely naturally on the fact that the base coordinate system is over a field.

4.2. A proof of the $8_{4}$ 'theorem' using projectivity groups over a field. This proof uses projectivities between the four lines $l_{i}$ of Section 4.1 .

A projectivity in the classical sense of von Staudt [22], who called it a throw (Wurf in German), is related to the bijection induced in the plane between the points on a line and the lines through a point, where the point and line are not incident. If we have a sequence of lines alternating with points then these projectivities may be multiplied, and they form a projectivity group when the sequences all start and end with the 
same line. It is a classical result that for any projective plane the same permutation group is obtained no matter what the starting line is. Also, the plane is Pappian (that is, coordinatized by a field) if and only if this group is sharply 3-transitive: every ordered set of three elements (points or lines) is mapped to any other ordered set of three elements by a unique member of the group. The group is then isomorphic to the classical group of $2 \times 2$ nonsingular matrices $\operatorname{PGL}(2, \mathbb{F})$, where $\mathbb{F}$ is the base field that coordinatizes the plane. See Strambach and Plaumann [16] and Coxeter [7] for the notation that we use below.

All this is known. What is less well known is that projectivity groups can be defined in a very similar way within any projective space. First, one starts with a skew line/secundum pair (a secundum being a subspace of dimension two less than the whole space). The hyperplanes containing the secundum are in natural bijective correspondence with the points on the line, and so a projectivity induced by incidence is obtained. Then by using a sequence of lines alternating with secunda, one obtains, in exactly the same way as in the plane, a projectivity group that maps the set of points on a line to itself (or dually the set of hyperplanes on a secundum). It is quite easy to calculate that the groups are isomorphic to the plane case. Thus, $\operatorname{PGL}(2, \mathbb{F})$ is obtained when the projective space is Pappian.

Now we can see that there is an unusual occurrence when the space has three dimensions: a line is also a secundum. Thus, given a sequence of an even number of lines, each skew to the next, and the last skew to the first, there are two ways of choosing them so that lines and secunda alternate. Hence, two different projectivities can be defined.

If $a, b$ and $c$ are three lines in three-dimensional space, with $a$ and $b$ skew, and $b$ and $c$ skew, then let us use the classical notation

$$
a \frac{b}{\pi} c
$$

to denote the projectivity with centre $b$ taking the points on $a$ to the points on $c$. Thus, if $P$ is any point on $a$, the image point on $c$ is the intersection of the plane $\langle P, b\rangle$ with $c$. Note that the inverse mapping is $c \frac{b}{\pi} a$.

For two skew lines $a$ and $b$ the projectivity with centre $b$ taking $a$ to itself, $a \frac{b}{\pi} a$, is trivial (the identity), but what happens if we have four lines?

Suppose that the space is Pappian and the coordinatizing field is algebraically closed (or at least extended enough). Now four general lines in a Pappian three-dimensional space have two transversals. One takes the unique quadric containing three of the lines and the intersection of the fourth line with the quadric is two points. Each of these points lies on a transversal. This means that the projectivities defined using a circuit of the four lines automatically have two fixed points. We need the following result.

LEMMA 4.1. Let $a, b, c, d$ be four mutually skew lines in Pappian three-dimensional space. Let $\pi$ be the projectivity

$$
a \frac{b}{\pi} c \frac{d}{\pi} a
$$


Then $\pi^{n}$ is the identity if and only if $P^{\pi^{n}}=P$ for any point $P$ on the line a with $P^{\pi} \neq P$.

ProOf. Suppose that $P^{\pi^{n}}=P$. We have noted already that $\pi$ has two fixed points. Thus, $\pi^{n}$ has at least three fixed points. Since the group of projectivities is sharply 3-transitive the identity projectivity is the only member of the group that fixes three points. Hence, $\pi^{n}$ is the identity. The converse is obvious.

REMARK. Lemma 4.1 applies to any Pappian projective space (not just those 'extended enough'), because an appropriate field and, hence, geometrical extension of the space may be made.

Consider the tetrahedron $P_{1}, P_{2}, P_{3}, P_{4}$ of our $8_{4}$ configuration. The projectivity

$$
\rho:=l_{1} \frac{l_{3}}{\pi} l_{2} \frac{l_{4}}{\pi} l_{1}
$$

defined by the 'circuit' $l_{1} . l_{3} . l_{2} \cdot l_{4}$ (starting and ending with the line $l_{1}$ ) takes $P_{1}$ via the plane $h_{5}=\left\langle P_{1}, l_{3}\right\rangle$ to the point $P_{2}=h_{5} \cap l_{2}$ and then via the plane $h_{6}=\left\langle P_{2}, l_{4}\right\rangle$ to the point $P_{3}$. Then applying $\rho$ again, $P_{3}$ goes via $h_{7}$ to $P_{4}$, and then via $h_{8}$ back to $P_{1}$. From Lemma 4.1, $\rho^{2}$ is the identity.

Similarly, consider the tetrahedron $P_{5}, P_{6}, P_{7}, P_{8}$, and the projectivity $\phi$ defined by the circuit $l_{3} \cdot l_{2} \cdot l_{4} \cdot l_{1}$. This also has order two and clearly the meaning of the $8_{4}$ 'theorem' is that $\rho$ has order two if and only if $\phi$ has order two.

To show this geometrically we consider the tetrahedron with (point) vertices $h_{1}, h_{2}$, $h_{3}, h_{4}$. Since $h_{1}, h_{2}, P_{5}, P_{6}$ and $P_{8}$ are coplanar, the line $h_{1} . h_{2}$ intersects $l_{4}=P_{6} . P_{8}$. Similarly $h_{2} . h_{3}$ intersects $l_{3}, h_{3} . h_{4}$ intersects $l_{4}$, and $h_{4} \cdot h_{1}$ intersects $l_{3}$. Thus we see that $\rho$ takes $h_{1}$ to $h_{3}$, and it then takes $h_{3}$ back to $h_{1}$. Hence the fact that $\phi$ has order 2 implies that $\rho^{2}$ takes $h_{1}$ to itself. Hence $\rho$ has order 2 if $\phi$ has order 2 . Thus we indeed have a 'theorem' in this 84 configuration.

REMARK. It is possible to use matrix calculations to verify this result about projectivities. Briefly, we can assume that the points on the line $l_{1}$ have homogeneous coordinates $(0,0, x, y)$, the points on the line $l_{2}$ have coordinates $(x, y, 0,0)$, the points on the line $l_{3}$ have coordinates $(x, y, x, y)$, and the points on the line $l_{4}$ have coordinates $(x, y, a, b)$, where $(a, b)=(x, y) A$, for some nonsingular $2 \times 2$ matrix $A$ over $\mathbb{F}$. We can assume that $A \neq k I$ as otherwise $l_{4}$ is in the regulus determined by the other $l_{i}$, and in this case $\rho$ would be equal to the identity. Then it is easy to check that $\rho$ has order two if and only if $A^{2}=k I(k \neq 0)$ if and only $\operatorname{tr}(A)=0$. One can then verify by using these matrices that the projectivity $\phi$ has order that. See Fishback $[9, \S 8.6]$ for further information and some history of projectivities of order two, that is, involutions on the line (in the case of the real numbers).

REMARK. The relationship between the four lines $l_{i}$ is actually one about pairs of pairs of lines: that is, $\left\{\left\{l_{1}, l_{2}\right\},\left\{l_{3}, l_{4}\right\}\right\}$. For the projectivity $l_{1} \frac{l_{3}}{\pi} l_{2} \frac{l_{4}}{\pi} l_{1}$ is equal to its 
inverse $l_{1} \frac{l_{4}}{\pi} l_{2} \frac{l_{3}}{\pi} l_{1}$ (they have order two), and also

$$
l_{3} \frac{l_{2}}{\pi} l_{4} \frac{l_{1}}{\pi} l_{3}=l_{3} \frac{l_{1}}{\pi} l_{4} \frac{l_{2}}{\pi} l_{3} \text {. }
$$

REMARK. An easy way to construct such pairs of pairs of lines is to start with a general tetrahedron $T$ in three-dimensional space. Let $\left\{l_{1}, l_{2}\right\}$ be opposite edges of $T$. Then let $l_{3}$ be any line joining any point on a further edge of $T$ with any point on its opposing edge. There are two remaining edges of $T$, which also form an opposite pair. Finally, $l_{4}$ can be chosen to be any line that joins points on these last edges.

4.3. A proof using homogeneous coordinates. In addition to the above more geometrical proof, we now give a completely algebraic one that only uses homogeneous coordinates.

PROOF. Let the first tetrahedron be coordinatized by the unit vectors as follows: $P_{1}=(1,0,0,0), P_{2}=(0,1,0,0), P_{3}=(0,0,1,0), P_{4}=(0,0,0,1)$. Let the second tetrahedron be $P_{5}=\left(a_{1}, a_{2}, a_{3}, a_{4}\right), P_{6}=\left(b_{1}, b_{2}, b_{3}, b_{4}\right), P_{7}=\left(c_{1}, c_{2}, c_{3}, c_{4}\right)$, $P_{8}=\left(d_{1}, d_{2}, d_{3}, d_{4}\right)$, where $a_{i}, b_{i}, c_{i}, d_{i} \in \mathbb{F}$.

Then the eight plane conditions become

$$
\left|\begin{array}{ll}
a_{1} & c_{1} \\
a_{2} & c_{2}
\end{array}\right|=\left|\begin{array}{ll}
a_{3} & c_{3} \\
a_{4} & c_{4}
\end{array}\right|=\left|\begin{array}{ll}
b_{1} & d_{1} \\
b_{4} & d_{4}
\end{array}\right|=\left|\begin{array}{ll}
b_{2} & d_{2} \\
b_{3} & d_{3}
\end{array}\right|=0
$$

and

Thus

$$
\left|\begin{array}{ll}
a_{1} & b_{1} \\
a_{3} & b_{3}
\end{array}\right|=\left|\begin{array}{ll}
a_{2} & d_{2} \\
a_{4} & d_{4}
\end{array}\right|=\left|\begin{array}{ll}
c_{1} & d_{1} \\
c_{3} & d_{3}
\end{array}\right|=\left|\begin{array}{ll}
c_{2} & b_{2} \\
c_{4} & b_{4}
\end{array}\right|=0 .
$$

$$
\begin{gathered}
a_{1} c_{2}-a_{2} c_{1}=a_{4} c_{3}-a_{3} c_{4}=b_{4} d_{1}-b_{1} d_{4}=b_{3} d_{2}-b_{2} d_{3}=0, \text { and } \\
a_{1} b_{3}-a_{3} b_{1}=a_{4} d_{2}-a_{2} d_{4}=c_{3} d_{1}-c_{1} d_{3}=c_{2} b_{4}-c_{4} b_{2}=0 .
\end{gathered}
$$

Now by multiplying the first monomials in the first four expressions above together, and also the second monomials paired with them, the first four of these determinant conditions imply that

$$
a_{1} a_{4} b_{4} b_{3} c_{3} c_{2} d_{2} d_{1}=a_{3} a_{2} b_{2} b_{1} c_{1} c_{4} d_{4} d_{3},
$$

which is the same condition implied by the last four determinant conditions above. Thus, we see that any seven of the conditions imply the final one, and the 84 'theorem' is proved in an algebraic fashion.

\section{A 94 'theorem' for three-dimensional space (over a field or skew-field)}

Let $l$ be a line of three-dimensional space and let $A, B, C$ be three distinct points on $l$. Let $\alpha_{1}, \alpha_{2}$, and $\alpha_{3}$ be three distinct planes through $l$. Let $T_{1}=\left\{P_{11}, P_{12}, P_{13}\right\}$ in $\alpha_{1}, T_{2}=\left\{P_{21}, P_{22}, P_{23}\right\}$ in $\alpha_{2}, T_{3}=\left\{P_{31}, P_{32}, P_{33}\right\}$ in $\alpha_{3}$ be triangles of points in these planes such that for all $1 \leq i \leq 3, P_{i 1} . P_{i 2}$ is a line through $A, P_{i 1} . P_{i 3}$ is a line through $B$, and $P_{i 2} . P_{i 3}$ is a line through $C$. See Figure 2. 


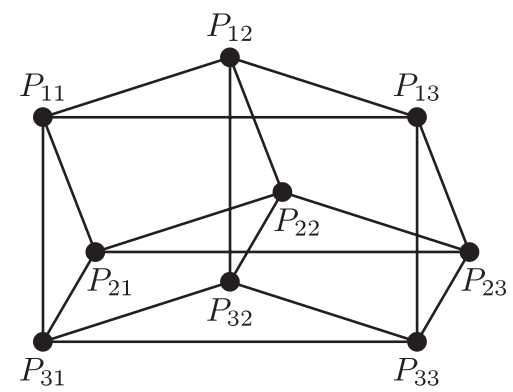

FiguRE 2. The $9_{4}$ 'theorem'.

By the three-dimensional Desargues theorem it follows that the triangles $T_{1}$ and $T_{2}$ are in perspective from a point $D$ : that is, the corresponding points $P_{1 j}$ and $P_{2, j}$ are collinear with $D$, for $1 \leq j \leq 3$. Similarly, the triangles $T_{1}$ and $T_{3}$ are in perspective from a point $E$, while the triangles $T_{2}$ and $T_{3}$ are in perspective from a point $F$.

Further, using Desargues theorem again it is possible to show that the three points $D, E$ and $F$ are collinear. (See the following remark.) There are nine planes defined as follows: these each contain one of the three collinear points $A, B, C$, and one of the other three collinear points $D, E, F$. Each of these planes also contains four points $P_{i j}$. For example, the plane containing $A$ and $D$ also contains $P_{11}, P_{12}, P_{21}$ and $P_{22}$.

The combinatorial configuration of the nine points $P_{i j}$ and the nine planes is the $9_{4}$ 'theorem' to be discussed in this section.

REMARK. The textbook by Fishback [9, §5.1, Exercise 9] asks the reader to prove (using Desargues theorem) the result: 'If three triangles are Desarguean in pairs with a common axis of perspectivity, the centers of perspectivity are concurrent'. In $[9, \S 5.1$, Exercise 10] there is the dual question, reversing the roles of centres and axes. Clearly this plane 'theorem' is closely related to the $9_{4}$ 'theorem'.

If we consider the graph defined by the nine points $P_{i j}$ with edges $\left\{P_{i u}, P_{i v}\right\}$ $(1 \leq i \leq 3,1 \leq u<v \leq 3)$ and $\left\{P_{i u}, P_{j u}\right\}(1 \leq i<j \leq 3,1 \leq u \leq 3)$, it is the graph on nine vertices that is the Cartesian product of the complete graph on three vertices, $K_{3}$, with itself. Equivalently, it is the line graph of the complete bipartite graph $K_{3,3}$.

REMARK. This $9_{4}$ configuration is geometrically self-dual. Since it is associated with the graph $K_{3,3}$, it has an automorphism group of order $3 ! \times 3 ! \times 2=72$. This group of order 72 thus appears as the subgroup of the symmetric group $S_{6}$ that preserves a subset of size three, or takes it to its complementary subset of size three. It is well known that $\operatorname{PSp}(4,2) \cong S_{6}$, and this leads us to a geometrical representation of the configuration and its group, as considered in the following remark.

REMARK. When $q$ is even, the projective symplectic group $\operatorname{PSp}(4, q)$ has order $q^{4}\left(q^{4}-1\right)\left(q^{2}-1\right)$, which is 720 when $q=2$. Also $\operatorname{PSp}(4,2)$ is the automorphism group of the linear complex $W(2)$ of 15 points and 15 lines embedded in the finite projective three-dimensional space $\operatorname{PG}(3,2)$ over $\mathrm{GF}(2)$. There are 35 lines in $\mathrm{PG}(3,2)$. 
If we consider the symplectic polarity $\omega$ corresponding to the linear complex in $\operatorname{PG}(3,2)$ then there are $(35-15) / 2=10$ pairs of conjugate lines $\left\{l, l^{\omega}\right\}$ such that $l \neq$ $l^{\omega}$. It may be ascertained that if any of these pairs of lines, and the six points on them, and dually the six planes through the lines, are deleted from $\operatorname{PG}(3,2)$, then the remaining configuration contains the 94 'theorem'. (The incidences that are a point $P$ on its polar plane $P^{\omega}$ have to be disregarded.) Since $72=720 / 10$, the group of automorphisms of the $9_{4}$ configuration occurs as a subgroup of $\operatorname{PSp}(4,2)$, and it is the subgroup that fixes any set of two conjugate lines $l \neq l^{\omega}$ of the symplectic polarity $\omega$ of $\operatorname{PG}(3,2)$.

LEMMA 5.1. In three-dimensional space consider three points of a triangle $A_{1}, A_{2}, A_{3}$ in a plane, and three points in a triangle $B_{1}, B_{2}, B_{3}$ on another plane, such that the three sets of four points, $\left\{A_{1}, A_{2}, B_{1}, B_{2}\right\},\left\{A_{1}, A_{3}, B_{1}, B_{3}\right\}$, $\left\{A_{2}, A_{3}, B_{2}, B_{3}\right\}$ are coplanar. Then the two triangles are in perspective from $a$ point $X$. That is, $A_{i}, B_{i}, X$ are collinear for $1 \leq i \leq 3$.

Proof. The three lines joining $A_{i}$ with $B_{i}$ are pairwise in planes, and so they intersect nontrivially. However, three lines in three-dimensional space with this property must be either coplanar, or concurrent in a point $X$. If they were coplanar, the triangles would be 'flattened out' into lines. This is not the case, and so the lines are concurrent.

There are various geometric observations that help with the synthetic proof of the $9_{4}$ 'theorem'. First, we rely on Lemma 5.1. Referring to that lemma, a prism is a certain figure of six points defined by three planes on its sides.

There are six triangles in the 94 configuration, and also six prisms. Each plane of four points is in two prisms, each triangle is in two prisms, and each prism contains two triangles.

If we do not know whether a certain four-point plane is satisfied in the configuration, then since the plane is in two prisms we do know that we have four prisms satisfied. Then Lemma 5.1 tells us that various triangles are in perspective from four points. By using Desargues theorem four times we see how there are two further points of perspective, yielding the fact that the last plane of four points is satisfied. Thus, the resulting configuration has six further points of perspective, grouped into two lines of three points each.

After these observations we present a brief synthetic proof of the $9_{4}$ 'theorem'.

PROPOSITION 5.2. The $9_{4}$ configuration is a 'theorem' in projective space of three dimensions over a field or skew-field.

PROOF. Using a similar notation to above let the configuration have the nine points $P_{i j}, 1 \leq i \leq 3,1 \leq j \leq 3$. Let the following be eight planes:

$$
\begin{aligned}
\pi_{33}:=\left\{P_{11}, P_{21}, P_{12}, P_{22}\right\}, & \pi_{32}:=\left\{P_{11}, P_{21}, P_{13}, P_{23}\right\}, \\
\pi_{13}:=\left\{P_{21}, P_{31}, P_{22}, P_{32}\right\}, & \pi_{11}:=\left\{P_{22}, P_{32}, P_{23}, P_{33}\right\}, \\
\pi_{12}:=\left\{P_{21}, P_{31}, P_{23}, P_{33}\right\}, & \pi_{23}:=\left\{P_{11}, P_{31}, P_{12}, P_{32}\right\}, \\
\pi_{22}:=\left\{P_{11}, P_{31}, P_{13}, P_{33}\right\}, & \pi_{21}:=\left\{P_{12}, P_{32}, P_{13}, P_{33}\right\} .
\end{aligned}
$$


We have to show that the four points $P_{12}, P_{22}, P_{13}, P_{23}$ lie on a plane $\pi_{31}$. Using Lemma 5.1 and planes $\pi_{12}, \pi_{22}, \pi_{32}$, the two triangles $P_{11}, P_{21}, P_{31}$ and $P_{13}, P_{23}, P_{33}$ are in perspective from a point $\alpha_{1}$. Similarly, using planes $\pi_{13}, \pi_{23}, \pi_{33}$, the triangles $P_{11}, P_{21}, P_{31}$ and $P_{12}, P_{22}, P_{32}$ are also in perspective from a point $\alpha_{2}$. Using planes $\pi_{21}, \pi_{22}, \pi_{23}$, the triangles $P_{11}, P_{12}, P_{13}$ and $P_{31}, P_{32}, P_{33}$ are in perspective from a point $\omega_{1}$. Using planes $\pi_{11}, \pi_{12}, \pi_{13}$, the triangles $P_{21}, P_{22}, P_{23}$ and $P_{31}, P_{32}, P_{33}$ are in perspective from a point $\omega_{2}$.

Consider the line $l:=\omega_{1} . \omega_{2}$ generated by $\omega_{1}$ and $\omega_{2}$. Using the perspectivity from $\alpha_{1}$, we see that the point of intersection of lines $m:=P_{11} \cdot P_{21}$ and $n:=P_{13} . P_{23}$ is on $l$, that is, $n$ is on the intersection $X$ of $m$ with $l$, while using the perspectivity from $\alpha_{2}$, we also see that the point of intersection of the lines $m$ and $r:=P_{12} . P_{22}$ is on $l$, that is, $r$ is on $X$. Thus, we see that $m, n$ and $r$ are concurrent with $X$ on $l$. In particular, the lines $n$ and $r$ meet at this point. Since these lines contain the points $P_{12}, P_{22}, P_{13}, P_{23}$, they are coplanar and the plane may be labelled $\pi_{31}$.

\section{Minimum-energy configurations of electrons}

Thomson studied configurations of electrons arranged on a sphere in threedimensional Euclidean space. Since electrons repel each other according to an inverse square law if a collection of $n$ electrons are 'thrown' randomly onto a sphere then they will automatically reconfigure into a minimum energy configuration. For small numbers of electrons (up to 15) the pattern is unique; that is, the local energy minimum is also global. See [3].

EXAMPLE 1. For three electrons the minimum energy occurs when they are equally spaced on a great circle (for example, the equator). When there are four electrons they form a tetrahedron on the sphere. Six electrons form an octahedron.

EXAMPlE 2 (The 84 'theorem'). When there are eight electrons they form two squares on two parallel planes. Note that this is not a cube. Instead, the second square is rotated at $45^{\circ}$ to the first, so that the diagonals of one square are parallel with the sides of the other. This means that there are eight more planes defined, each containing a side of one square and a diagonal of the other. Clearly the eight points and these latter eight planes form the 84 configuration of Section 4 , in a special case where the two tetrahedra are collapsed into parallel planes.

EXAMPLE 3 (The 94 'theorem'). When there are nine electrons we again have a connection with an $n_{k}$ 'theorem'. In this case the minimum energy occurs in a configuration having three equilateral triangles in parallel planes on the sphere. The 'top' and 'bottom' triangles are oriented in the same way, vertically below each other with respect to the poles of the sphere, while the 'middle' triangle on the 'equator' is rotated at $180^{\circ}$ with respect to the other two. However, the sides of all three triangles still occur in three parallel classes. This configuration is seen to be another case of the $9_{4}$ 'theorem' of Section 5. 


\section{Forbidden minors in graph/matroid theory}

Many 'forbidden minor' characterizations of graphs and matroids correspond to certain lists of $n_{k}$ 'theorems'. 'Minors' are the matroid equivalent of submatrices of matrices over a field, the columns of which coordinatize configurations in space. For an elementary introduction to matroids and graphs see Wilson [21]. Some examples follow.

We denote the Fano matroid (that is, the projective plane of order two) by $F$, and the dual of a matroid $M$ by $M^{*}$. 'Binary' matroids are those representable over $\mathrm{GF}(2)$.

EXAMPLE 4. A matroid is binary if and only if it has no minor isomorphic to the fourpoint line; that is, the rank-two matroid having four points, every pair independent.

REMARK. The 'Ursatz' 22 'theorem' also has four points (actually two of them are 'dual' points) on the line. However, this degenerate 'theorem' is about all of the points being the same if three pairs of them are equal.

EXAMPLE 5 (Tutte 1958 [17]). A matroid is regular if and only if it is binary and has no minor isomorphic to $F$ or $F^{*}$ if and only if it is unimodular.

Remark. The Fano matroid $F$ is the 73 'theorem' and $F^{*}$ is the $7_{4}$ 'theorem' for fields of characteristic two.

EXAMPLE 6 (Tutte 1959 [18]). A matroid is graphic (that is, it is the circuit matroid of a graph) if and only if it is binary and contains no minor isomorphic to $M^{*}\left(K_{5}\right)$, $M^{*}\left(K_{3,3}\right), F$ or $F^{*}$.

Remark. Here $M^{*}\left(K_{5}\right)$ and $M^{*}\left(K_{3,3}\right)$ are closely related to the $10_{7}$ and $9_{5}$ configurations, which are the complements of Desargues and $9_{4}$ 'theorems', while $F$ and $F^{*}$ are the $7_{3}$ and 74 'theorems'.

EXAMPLE 7. A graph (with nondirected edges) is nonplanar (every embedding into the Euclidean plane has an edge-crossing somewhere) if and only if it has a minor (a subgraph constructed by a sequence of deletions and contractions of edges) that is $K_{5}$ or $K_{3,3}$.

REMARK. The graph $K_{5}$ corresponds to the Desargues $10_{3}$ 'theorem' in threedimensional space. One way to construct such a configuration of ten points in the plane (over a skew-field) is to consider five independent points in four-dimensional space. There are ten subsets of two points from these five points, and these subsets generate ten lines intersecting a general hyperplane in the three-dimensional Desargues configuration. Any embedding of the Desargues configuration in the plane is obtained by a projection from the three-dimensional Desargues configuration from a point to the plane. Thus, the rank-four Desargues $10_{3}$ configuration is the circuit matroid of $K_{5}$.

EXAMPLE 8 . The graph $K_{3,3}$ corresponds to the $9_{4}$ 'theorem'. One way to construct the $9_{4}$ configuration in three-dimensional space is to consider six independent points in five-dimensional space. Split the six points into a partition of two subsets of three points each. The nine lines joining a point of one subset with a point from the 
other intersect a general hyperplane in the four-dimensional $9_{4}$ configuration. Then any embedding of the $9_{4}$ configuration in three-dimensional space is obtained by a projection of the four-dimensional configuration from a point to the three-dimensional space. In matroid theory the rank-five $9_{4}$ configuration represents the circuit matroid of $K_{3,3}$.

REMARK. It follows from Brylawski and Lucas [4] and White [20] that if a binary matroid is representable over a certain field, then it is uniquely representable, that is, up to homographies (linear collineations), in that projective (or affine) geometry. Since the circuit matroids of $K_{5}$ and $K_{3,3}$ are graphical, hence regular (representable over any field), unimodular (represented by a rational matrix with all subdeterminants 0 or \pm 1 ) and binary (embeddable in space over GF(2)), it follows that when they are embedded into three-dimensional and four-dimensional space (as the $10_{3}$ configuration and $9_{4}$ configuration, respectively), the embeddings are essentially unique. (In the same papers it is shown that any representation of any matroid over $\mathrm{GF}(3)$ is unique up to collineations.)

REMARK. If we have two tetrahedra in perspective from a point in three-dimensional space, then the corresponding sides of the tetrahedra intersect in six further points. In total there are

$$
1+4+4+6=15=\left(\begin{array}{l}
6 \\
2
\end{array}\right)
$$

points in the resulting configuration and, by using Desargues theorem six times, it has

$$
4+6+6+4=20=\left(\begin{array}{l}
6 \\
3
\end{array}\right)
$$

lines of three points each. It may be seen that it is obtained from a projection of the chords of the six points in five-dimensional space intersecting a four-dimensional space. Thus, the 94 configuration is a subconfiguration of the well-known 'two tetrahedra in perspective' configuration. In matroid theory this latter rank-five configuration of 15 points, 20 lines and 15 planes, represents the circuit matroid of $K_{6}$. Cayley and Veronese can be considered to be the originators of the study of configurations such as these. See Baker [1], Carver [5], Cayley [6], Hilbert and CohnVossen [12] and Veronese [19] for some of the history.

\section{Geometrical models}

It is possible to make nice models of the $8_{4}$ and $9_{4}$ configurations. We can make a model of the 94 configuration in Euclidean space as follows. Consider two regular triangular prisms: such a prism has three faces that are perfect squares, joined edgewise, and at the two ends there are equilateral triangles. Now glue the two prisms together at a pair of square faces so that one is twisted by $90^{\circ}$ with respect to the other. This gives a figure in space with $6+6-4=8$ vertices. Then the final point of the $9_{4}$ configuration is the centre point (centroid) with respect to the whole. The centroid 
will be in the plane common to both prisms. Join it to the remaining four vertices not in the common plane by lines of different colours. Each coloured line will be parallel to two other edges on the faces of the external figure. Colour these edges the same. Now we have the graph having 9 vertices and 18 edges, which is the Cartesian product of $K_{3}$ with itself, embedded in space. The nine planes of the $9_{4}$ configuration contain two edges from two parallel classes; that is, colours.

For a model of the 84 configuration consider a (regular) tetrahedron. Colour two opposite edges of the tetrahedron with the colour green. The remaining four edges we colour orange (they form a 4-cycle.) Now draw two lines between points on opposite sides of this orange 4-cycle. Colour these yellow. We have to construct a further four (red) lines (and eight points on them). Take any point on a green line. It is on a unique line that intersects both yellow lines. Colour this new line red. The red line intersects one of the yellow lines (the 'first') in a point which is on a further red line that intersects both yellow lines and the other green line. This further (second) red line contains an additional point on the second yellow line and this additional point is on a unique (third) red line that intersects the first yellow line and the first green line. The point on the first yellow line on this red line is on the unique (fourth) red line intersecting the second green line and the second yellow line. From the $8_{4}$ 'theorem' the fourth red line is concurrent with the second yellow and first red lines.

The eight points of the 84 configuration are the vertices of the original tetrahedron, together with the points of intersection of the $i$ th and $(i+1)$ th red lines, where $i$ is taken modulo four. The eight planes are in bijective correspondence with the remaining eight points of intersection between the two green, two yellow, four orange and four red lines. Each of these points is either on a pair of orange and yellow lines, or on a pair of red and green lines. Thus, the points of the $8_{4}$ configuration are on precisely three of the lines, while the planes correspond to points that are on precisely two of the lines.

\section{Summary}

At present we know of 'standard' $n_{k}$ 'theorems' that have parameters $7_{3}, 7_{4}, 8_{4}$ (twice), $9_{3}, 9_{4}, 9_{6}$ and $10_{3}$. The complementary $9_{5}$ and $10_{7}$ configurations are not embedded into their spaces in a standard way, in that either all of the points lie in a hyperplane, or all of the hyperplanes pass through a point. Also, the $7_{3}$ and its complementary 74 configurations are only 'theorems' for fields of characteristic two. The genesis of such 'theorems' may be considered to be the 'Ursatz' $m_{2}$ 'theorems' where $m \geq 2$. These have a Levi graph that is a $2 m$-cycle. However, these are not 'standard theorems' in that any embedding will have repeated points.

The searches we have made have been with a small computer and for the rational number field. It is possible that by searching over fields of positive characteristic and with a more powerful computer more $n_{k}$ 'theorems' could be found.

'Theorems' that are valid in all projective spaces (over a general skew field) have a different character to those that are only valid for just fields (or even fields of a certain characteristic). 'Theorems' valid for all projective spaces must be able to be proved 
using just synthetic methods and Desargues theorem, whereas 'theorems' for spaces over fields must use Pappus (or algebraic methods involving objects such as quadrics or determinants). See Hilbert [11].

Then there is still the aim to classify all $n_{k}$ 'theorems'. We speculate that it can be done using generalizations of von Staudt's projectivity groups to sequences of lines and secunda in higher-dimensional space.

The known $n_{k}$ 'theorems' that are also valid for skew fields (those that are provable by purely synthetic methods), that is, the $10_{3}$ and $9_{4}$ 'theorems', also have the property that they come by projection from a point of unique higher-dimensional (graphical) models in three and four dimensions, respectively. This leads us to speculate that there is a general connection between configurations that come by projection from unique higher-dimensional models, and 'theorems' of this kind. We have already noted at the end of Section 1 that the reason that the complementary $10_{7}$ and $9_{5}$ configurations degenerate is that they have this property.

\section{References}

[1] H. F. Baker, Principles of Geometry (Cambridge University Press, London, 1922, Second Edition 1929), Vol. I, Foundations; Vol. III, Solid Geometry.

[2] W. Bosma, J. Cannon and C. Playoust, 'The MAGMA algebra system I: the user language. Computational algebra and number theory', J. Symbolic Comput. 24 (1997), 235-265.

[3] K. S. Brown, Min-Energy Configuration of Electrons on a Sphere, http://www.mathpages.com/home/kmath005/kmath005.htm, (1994-2008).

[4] T. H. Brylawski and T. D. Lucas, Uniquely Representable Combinatorial Geometries, Atti dei Convegni Lincei, 17 (Accademia Nazionale dei Lincei, Rome, 1976), pp. 83-104 (in Proc. Vol. I of 'Colloquio Internazionale sulle Teorie Combinatorie', Roma, 1973).

[5] W. B. Carver, 'On the Cayley-Veronese class of configurations', Trans. Amer. Math. Soc. 6 (1905), 534-545.

[6] A. Cayley, 'Sur quelques théorèmes de la géométrie de position', J. Reine Angew. Math. [Crelle's J.] 31 (1846) (Collected Papers, vol. 1, p. 317).

[7] H. S. M. Coxeter, Introduction to Geometry, 2nd edn (John Wiley and Sons, New York, 1969).

[8] P. Dembowski, Finite Geometries, Ergebnisse der Mathematik und ihrer Grenzgebiete, Band 44 (Springer, Berlin, 1968).

[9] W. T. Fishback, Projective and Euclidean Geometry (John Wiley and Sons, New York, 1962).

[10] D. G. Glynn, 'A note on $N_{k}$ configurations and theorems in projective space', Bull. Aust. Math. Soc. 76 (2007), 15-31.

[11] D. Hilbert, Foundations of Geometry, 2nd edn. Translated from the 10th German edition by Leo Unger, Open Court, LaSalle, Ill. 1971.

[12] D. Hilbert and S. Cohn-Vossen, Anschauliche Geometrie, 2nd edn (Springer, Berlin, 1996) (translated as Geometry and the Imagination).

[13] F. W. Levi, Finite Geometrical Systems, Public Lecture Series, University of Calcutta, 1940 (University of Calcutta, Calcutta, 1942).

[14] B. McKay, NAUTY, The Graph Package (Australian National University, Canberra, 1984-2008), http://cs.anu.edu.au/ bdm/nauty/.

[15] A. F. Möbius, 'Kann von zwei dreiseitigen Pyramiden eine jede in Bezug auf die andere um- und eingeschrieben zugleich heissen?', J. Reine Angew. Math. 3 (1828), 273-278 (plus figures 1 and 2 in Tafel IV of Heft 3) (Gesammelte Werke, Vol. 1 (1885), 437-443).

[16] K. Strambach and P. Plaumann (eds), Geometry-Von Staudt's point of view, Proceedings of the NATO Advanced Study Institute, Bad Windsheim, 1980 (D. Reidel, Dordrecht, 1981).

[17] W. T. Tutte, 'A homotopy theorem for matroids. I, II', Trans. Amer. Math. Soc. 88 (1958), 144-174. 
[18] W. T. Tutte, 'Matroids and graphs', Trans. Amer. Math. Soc. 90 (1959), 527-552.

[19] A. Veronese, 'Behandlung der projectivischen Verhältnisse der Räume von verschiedenen Dimensionen durch das Princip des Projicirens und Schneidens' (Treatment of the projective relationships of spaces of different dimensions using the principle of projections and intersection), Math. Ann. 19 (1882), 161-234.

[20] N. White, 'The bracket ring of a combinatorial geometry. I', Trans. Amer. Math. Soc. 202 (1975), 79-95; 'The bracket ring of a combinatorial geometry. II: unimodular geometries', Trans. Amer. Math. Soc. 214 (1975), 233-248.

[21] R. J. Wilson, Introduction to Graph Theory, 3rd edn (Longman, New York, 1985).

[22] K. G. C. von Staudt, Geometrie der Lage (Bauer und Raspe, Nürnberg, 1847).

DAVID G. GLYNN, School of Mathematical Sciences, University of Adelaide, SA 5005, Australia

and

Current address: School of Computer Science, Engineering and Mathematics, Flinders University, GPO Box 2100, Adelaide SA 5001, Australia

e-mail: davidg@csem.flinders.edu.au 